\title{
ESTUDO DE EVENTOS AMPARADO EM MÉTRICAS CONTÁBEIS
}

\author{
EVENT STUDIES ON ACCOUNTING BASED MEASURES
}

\author{
Melisa Maia de Paula ${ }^{a}$; Jorge Vieira ${ }^{b}$ \\ ${ }^{a}$ Mestre em Ciências Contábeis pela Universidade do Estado do Rio de Janeiro - UERJ \\ Rio de Janeiro, RJ-Brasil; E-mail: melisamaia@yahoo.com \\ ${ }^{b}$ Professor Adjunto do Programa de Mestrado em Ciências Contábeis da Universidade do Estado do Rio de Janeiro - UERJ \\ Doutor em Controladoria e Contabilidade pela Universidade de São Paulo - FEA/USP \\ Rio de Janeiro, RJ-Brasil; E-mail: jorgevcosta@terra.com.br
}

\section{Resumo}

O estudo de eventos amparado em métricas contábeis caracteriza-se como aquele que busca investigar a existência de performances anormais após eventos corporativos. Esse tipo de estudo é observado em muitos trabalhos empíricos na área de Contabilidade e Finanças, podendo ser aplicado em muitos casos, tais como abertura de capital, fusões e aquisições, distribuição de dividendos, desdobramento de ações (split) e sua aquisição para manutenção em tesouraria, ofertas públicas, etc. Eventuais ganhos ou perdas detectados em um estudo de eventos sobre o retorno das ações podem ser corroborados com os ganhos operacionais reais, com o intuito de buscar fundamentos econômicos que justifiquem a variação no comportamento das ações. Este ensaio teórico tem como objetivo discutir as características intrínsecas dos modelos utilizados para se detectar performances operacionais anormais, haja vista a existência de uma considerável variedade de medidas contábeis e benchmarks que podem ser adotados, bem como testes estatísticos que podem ser aplicados, os quais podem vir a influenciar os resultados.

Palavras-chave: Estudo de eventos, Métricas contábeis; Performance operacional anormal.

\section{Abstract}

The event study on accounting based measures investigates the existence of abnormal corporate events performance (profits) after the occurrence of some abnormal corporate events. This type of study is documented in many empirical papers in the area of Accounting and Finance and it can be applied in many situations such as initial public offers - IPO's, mergers and acquisitions - M\&A's, payment of dividends, stock splits, share repurchases, seasoned equity offers - SEO's, etc. Occasional abnormal returns, detected by an event study on stock returns (market expectation), can be corroborated by real gains on operational performance (event study on accounting based measures), in order to identify the economic fundamentals that justify the behavior of the stocks (abnormal returns $x$ abnormal operational performances). This essay aims to explore the intrinsic characteristics of the models used to detect abnormal operational performances (event study on accounting based measures), since there is a considerable variety of accounting measures and benchmarks that can be used as well as statistical tests that can be applied, which can influence the results.

Keywords: Event studies; Accounting based measures; Abnormal operational performance

Recebido em: 06/09/2011 - Aceito em:10/05/2012 - 2a Revisão: 11/07/2012 - - $3^{\text {a }}$ Revisão: 16/08/2012 Sistema de Avaliação: Double Blind Review

Editor Científico: Amaury José Rezende 


\section{INTRODUÇÃO}

O estudo de eventos por retornos ao redor dos anúncios foi introduzido pelos trabalhos de Fama (1969) e Ball e Brown (1968) e pode ser compreendido como um método que consiste em verificar os impactos da divulgação de eventos econômicos específicos nos valores de mercado dos títulos das empresas. Este tipo de estudo tem sido amplamente utilizado no Brasil em muitos trabalhos científicos.

No entanto, Healy, Palepu e Ruback (1992) alertam para o fato da inabilidade dos estudos baseados nos preços das ações em determinar se os eventos, tais como fusões e aquisições, geram ganhos econômicos reais ou apenas se limitam a conhecer as expectativas do mercado.

Esta preocupação em verificar ganhos operacionais reais após eventos corporativos é observada em muitos trabalhos empíricos na área de Contabilidade e Finanças. Esse tipo de estudo pode ser aplicado em diversos casos, tais como abertura de capital, fusões e aquisições, distribuição de dividendos, desdobramento de ações (split) e sua aquisição para manutenção em tesouraria, ofertas públicas, etc. Eventuais ganhos ou perdas detectados em um estudo de eventos sobre o retorno das ações (expectativas do mercado) podem ser corroborados com os ganhos operacionais reais (estudo de eventos com métricas contábeis), com o intuito de buscar fundamentos econômicos que justifiquem a variação no comportamento das ações (retornos anormais x performances operacionais anormais).

Dessa forma, nas palavras de Costa Jr (2008), o estudo de eventos amparado em métricas contábeis poderia ser caracterizado como aquele que busca investigar se as expectativas do mercado, precificadas em um eventual retorno anormal positivo pós-anúncio, tiveram a contrapartida em uma eventual performance operacional positiva, após o ano em que uma determinada operação foi efetivada.

Neste contexto, a técnica do estudo de eventos pode ser definida da seguinte forma:

Em síntese, a técnica consiste em utilizar uma métrica contábil que meça a performance operacional da firma, por exemplo, o retorno operacional dos ativos da firma ou o fluxo de caixa operacional da firma escalonado pelos ativos totais, ajustá-la à performance operacional observada no conjunto de firmas que compõe o setor no qual está inserida a firma de interesse de estudo, confrontá-la em momentos distintos - antes e após o evento - e investigar se houve diferenças estatisticamente significativas, positivas ou negativas. (COSTA Jr e MARTINS, 2008, p. 8).

Diante do exposto, este trabalho tem como objetivo discutir as características intrínsecas dos modelos que utilizam medidas baseadas nas demonstrações contábeis para detectar os efeitos de decisões corporativas na performance operacional das empresas.

\section{ESTUDOS DE EVENTOS AMPARADOS EM MÉTRICAS CONTÁBEIS NA LITERATURA INTERNACIONAL}

Na literatura internacional, muitas pesquisas empíricas focam o desempenho operacional 
das firmas quando ocorrem eventos corporativos. Porém, existe uma considerável variedade de medidas contábeis e benchmarks que podem ser adotados, bem como testes estatísticos que podem ser aplicados a fim de detectar as performances operacionais anormais, cujas escolhas podem vir a influenciar os resultados.

Um trabalho de referência na literatura internacional é o de Healy, Palepu e Ruback (1992). Estes autores examinaram a performance operacional das cinquenta maiores firmas envolvidas em operações de fusões e aquisições nos EUA, no período de 1979 a 1983.

O modelo utilizado por estes autores é chamado por Ghosh (2001) como modelo do intercepto. Por este modelo, ao comparar a pós-performance (posterior à fusão e à aquisição) com a pré-performance pro forma (onde são agregados dados contábeis das empresas alvo e adquirente), obtêm-se as mudanças no desempenho operacional.

Porém, para comparar o desempenho das empresas antes e depois de um determinado evento, é necessário, primeiro, determinar qual seria o desempenho esperado na ausência do evento. Assim, o desempenho das firmas da amostra pós-evento precisa ser comparado ao desempenho de um determinado conjunto de firmas, chamado de grupo de controle ou grupo de comparação, que servirá como benchmark do desempenho operacional esperado depois do evento. A diferença entre o desempenho observado das firmas da amostra e do grupo de controle (desempenho esperado) é chamado de desempenho operacional anormal.

Para Healy, Palepu e Ruback (1992) deve-se usar como benchmark o desempenho setorial, "performance ajustada ao setor", com o objetivo de controlar os efeitos das mudanças econômicas. Mikkelson, Partch e Shah (1997) também corroboram este argumento.

A "performance ajustada ao setor" é obtida diminuindo-se do valor obtido para a empresa amostrada, a mediana/média do desempenho do setor.

Os resultados do trabalho de Healy, Palepu e Ruback (1992) apontam que as empresas fusionadas cresceram no período, com relação ao setor industrial a que pertencem, e que este crescimento é decorrente do aumento da produtividade dos ativos.

Os autores também evidenciam uma relação entre a medida de desempenho estabelecida e aquelas baseadas no comportamento do mercado de ações. Isto quer dizer que, em parte, a valorização que ocorre nas ações deriva da antecipação dos agentes econômicos dos ganhos reais resultantes da fusão. Dessa forma, Healy, Palepu e Ruback (1992) comprovam a existência de um elo de ligação entre as expectativas do mercado e os ganhos reais econômicos. Outro trabalho importante na literatura internacional é o de Jain e Kini (1994) que buscam investigar as mudanças nas performances operacionais das firmas que realizam abertura de capital - IPO. Este foi o primeiro estudo a considerar as mudanças operacionais pós-IPO de uma forma geral. Os autores analisaram 682 empresas que realizaram IPO entre 1976 e 1988 nos EUA e analisaram a performance operacional utilizando duas medidas de desempenho: Retorno Operacional dos Ativos - ROA e Fluxo de Caixa Operacional/Ativo Total.

Jain e Kini (1994) estabeleceram dois modelos para verificar as variações ocorridas na performance operacional antes e depois do IPO. Um modelo que considera a variação no desempenho operacional como a mediana nos níveis de mudança (sem ajuste - unadjusted) e outro que considera a variação no desempenho operacional ajustado à indústria. 
Utilizando os modelos estabelecidos, os autores realizaram testes estatísticos, teste Wilcoxon two-tailed signed rank tests, para verificar se a variação ou mudança na performance operacional das firmas que realizaram IPO é significativamente diferente de zero.

Jain e Kini (1994) chegaram à conclusão de que as firmas exibem um desempenho operacional inferior no período pós-IPO, apesar do aumento das vendas e das despesas de capital. Os autores justificam que esse declínio é consistente com o seguinte: as empresas costumam gerenciar resultados e apresentar bons desempenhos no período pré-IPO, aproveitando os bons momentos de performance operacional para fazer abertura de capital, porém estes bons momentos não se sustentam no futuro.

Em se tratando de fusões e aquisições, Ghosh (2001) constatou que as empresas no período pré-evento apresentam desempenho superior ao do setor, o que explica a característica tendenciosa dos modelos para detectar retornos anormais que não levam em consideração o desempenho das empresas no período pré-evento corporativos.

A crítica de Ghosh (2001) encontra sustentação no efeito "reversão à média de dados contábeis". A "reversão à média" da rentabilidade é um pressuposto econômico de que, em um ambiente competitivo, a taxa de retorno dos investimentos tende a se igualar em todos os setores. Este efeito foi comprovado empiricamente por Fama e French (2000): os autores confirmaram os argumentos econômicos de que a rentabilidade se reverte à média e que a taxa de reversão é maior, quanto mais distante estiver a rentabilidade da sua média.

Barber e Lyon (1996) explicam que este efeito ocorre quando existem componentes transitórios no resultado operacional, implicando em receitas ou despesas não recorrentes, advindos em grande parte de mudanças de práticas contábeis, gerenciamento de resultados, ou forças econômicas subjacentes, como a sazonalidade da demanda, . Com o tempo, à medida que os componentes transitórios desaparecem, o resultado operacional tende a retornar à média populacional. Assim, o controle por nível de pré-performance tem o objetivo de isolar os efeitos transitórios não relacionados ao evento.

Na intenção de contribuir para as discussões acima, e em crítica ao trabalho de Healy, Palepu e Ruback (1992), Ghosh (2001) afirma que podem ocorrer interferências na regressão, o que conduz a resultados tendenciosos quando se usa empresas medianas representativas da indústria. $\mathrm{O}$ autor explica que os erros de mensuração raramente são aleatórios, pois as empresas fusionadas costumam ter bons desempenhos antes das aquisições, como já comprovado em trabalhos anteriores como o de Mork et al. (1990). Neste contexto, Ghosh (2001) empreende estudo para verificar se o fluxo de caixa operacional aumenta depois de aquisições corporativas, utilizando um delineamento de pesquisa que considera um desempenho superior pré-aquisição.

A amostra foi formada por 315 pares de firmas que tiveram o processo de fusão completo entre 1981 e 1995. E a métrica contábil utilizada foi o fluxo de caixa operacional tal como definido em Healy, Palepu e Ruback (1992).

Ghosh (2001) enfatiza que tanto os modelos de intercepto (como o de Healy, Palepu e Ruback, 1992) quanto os modelos que fazem uma simples comparação entre os fluxos de caixa ajustados à indústria (change models) pré e pós-aquisições, possuem problemas econométricos advindos dos seguintes fatores:

a) erros de mensuração; 
b) pré-performance das empresas fusionadas superior à mediana da indústria; e

c) diferenças de performance entre as companhias fusionadas e a mediana das empresas da indústria com base em fatores permanentes ou temporários, que levam a performances anormais.

Para evitar os erros dos estudos anteriores, o autor comparou a pré e pós-performance das companhias fusionadas em relação às empresas de controle combinadas por pré-performance e tamanho.

Ghosh (2001) utilizou a mesma metodologia de Healy, Palepu e Ruback (1992), chegando às mesmas conclusões de que o desempenho operacional das empresas aumenta depois de processos de fusão. Porém, ao combinar as firmas por tamanho e performance, modelo de Barber e Lyon (1996), o autor não encontrou nenhuma evidência de melhoria do desempenho operacional. Verifica-se que, dependendo do modelo utilizado, os resultados podem ser bem diferentes.

Este problema também é observado em relação a outros tipos de eventos, como a abertura inicial de capital - IPO. Jain e Kini (1994), por meio do modelo que utiliza como benchmark a indústria, constataram piora no pós-desempenho operacional. Biral (2010) replicou este estudo no Brasil, usando como benchmark indústria e tamanho, e não conseguiu concluir com significância estatística se o IPO é capaz de alterar o desempenho operacional das empresas. Por outro lado, González e Farias (2009) controlaram por indústria, tamanho e pré-performance, e verificaram que, no Chile, as empresas que realizam IPO apresentam uma melhora no desempenho operacional. Esses autores revelam que os resultados do Chile são diferentes dos encontrados no resto do mundo devido às especificidades no mercado e nas empresas chilenas, como competitividade e alta retenção de capital pelas empresas que realizam IPO.

Um estudo que evidencia de forma clara as diferenças que podem ser geradas nos resultados, dependendo do modelo utilizado, é o de Guo, Hotchkiss, e Song (2009). Neste trabalho são verificadas 192 aquisições alavancadas, entre 1990 e 2006 nos EUA. Os autores utilizam três tipos de benchmark, encontrando resultados diferentes para cada um deles: no modelo sem ajuste (unadjusted), o desempenho encontrado foi negativo; no modelo ajustado à indústria, os ganhos não foram significativos, e, por fim, no modelo ajustado à pré-performance, os ganhos são significativos, porém abaixo dos apresentados em estudos anteriores.

Da mesma forma que nos exemplos apresentados acima, outros estudos de eventos amparados em métricas contábeis apresentam divergências em relação aos modelos para detectar retornos operacionais anormais e resultados alcançados.

Três questões são então aqui levantadas para justificar a variedade de resultados na análise do desempenho operacional pós-eventos: cada evento possui sua particularidade e embasamento teórico para os resultados esperados; os resultados irão depender do modelo utilizado, conforme já apontado por Barber e Lyon (1996) e Ghosh (2001); e, por fim, os resultados podem variar de acordo com os mercados analisados. 


\section{TESTANDO A CAPACIDADE DOS MODELOS PARA DETECTAR DESEMPENHO OPERACIONAL ANORMAL}

Conforme observam Baber e Lyon (1996), existe uma considerável variação nas medidas contábeis e nos testes estatísticos utilizados para se detectar performances anormais. Ainda, conforme apresentado anteriormente, resultados diferentes podem ser alcançados dependo do modelo utilizado.

Com o objetivo de avaliar os métodos usados em estudos de eventos que empregam métricas contábeis de desempenho operacional, Barber e Lyon (1996) realizam pesquisas para analisar o impacto de escolhas em relação a três pontos:

a) medidas de performance baseada em métricas contábeis;

b) modelo de expectativa da performance operacional - benchmark; e

c) teste estatístico.

A amostra utilizada é composta por aproximadamente 30.000 empresas listadas na New York Stock Exchange - NYSE, entre 1977 e 1992.

Os grupos de controle ou comparação são formados com base em variáveis definidas nas características da empresa componente da amostra no período pré-performance. São definidos quatro benchmarks:

1. código de dois dígitos da base de dados "Compustat" - indústria. A justificativa é de que parte das variações na performance operacional pode ser justificada pelas variações econômicas do setor a que pertence a empresa;

2. código de quatro dígitos da base de dados "Compustat"- indústria;

3. indústria e tamanho; justifica a utilização deste critério no estudo de Fama e French (1995) que documentou que as pequenas empresas, na média, possuem menor lucro por patrimônio líquido do que as grandes, e pelos trabalhos de: Kaplan (1989), Denis e Denis (1993) e DeGeorge e Zeckhauser (1993). O filtro utilizado selecionou empresas que possuíam entre $70 \%$ e $130 \%$ do valor contábil dos ativos da firma $i$.

4. indústria e desempenho passado; utilizando a pré-performance da indústria é possível controlar a tendência de reversão à média. $\mathrm{O}$ filtro para composição dos grupos são as firmas que possuem entre $90 \%$ a110\% do valor do Retorno sobre os Ativos da firma i.

Com base nos grupos de comparação acima mencionados, Barber e Lyon (1996) desenvolvem os modelos de expectativas de performance (performance na ausência do evento) que são obtidos da seguinte forma:

$$
\mathrm{E}(\mathrm{Pit})=\mathrm{PI}_{\mathrm{it}}^{\mathrm{j}}, \mathrm{j}=1,4
$$

Onde, a expectativa de performance da empresa i no tempo t - E(Pit) é igual à performance 
da indústria (considerando a mediana da indústria) no tempo t $-\mathrm{PI}^{\mathrm{j}}{ }_{\mathrm{it}}$, para cada um dos quatro grupos de comparação (j) definidos anteriormente.

Esses quatro primeiros modelos são chamados de modelos no nível de desempenho industrial - level models.

Quando se acrescenta a questão das mudanças nas performances, são fornecidos mais quatro modelos:

$$
\begin{gathered}
\mathrm{E}(\mathrm{Pit})=\mathrm{Pi} \mathrm{t}-1+\Delta \mathrm{PI}^{\mathrm{j}}{ }_{\mathrm{it}} \text {, sendo } \mathrm{j}=1,4 \text { e onde: } \\
\Delta \mathrm{PI}^{\mathrm{j}}{ }_{\mathrm{it}}=\mathrm{PI}_{\mathrm{t}}^{\mathrm{j}}-\mathrm{PI}^{\mathrm{j}}{ }_{\mathrm{t}-1}
\end{gathered}
$$

Esses últimos modelos são chamados de modelos com desempenho defasado da empresa e mudança no desempenho industrial, ou simplesmente modelos de mudanças - change models.

O nono e último modelo considera que o desempenho da empresa é uma resposta ao seu passado, sem considerar o desempenho industrial:

$$
\mathrm{E}(\mathrm{Pit})=\mathrm{Pi} \mathrm{t}-1
$$

Nos modelos acima, $\mathrm{PI}^{\mathrm{j}}{ }_{\mathrm{it}}=$, a mediana da indústria é utilizada.

A partir dos modelos de expectativa de performance, Barber e Lyon (1996) definiram o desempenho anormal - $\mathrm{AP}_{\mathrm{it}}$ - da empresa $i$ no tempo $t$, como o desempenho no ano $t$ menos o desempenho esperado:

$$
\mathrm{AP}_{\mathrm{it}=} \mathrm{P}_{\mathrm{it}}-\mathrm{E}\left(\mathrm{P}_{\mathrm{it}}\right)
$$

Sendo $\mathrm{P}_{\mathrm{it}}=\mathrm{ROA}_{\mathrm{it}}$ e $\mathrm{E}\left(\mathrm{P}_{\mathrm{it}}\right)=$ cada um dos 9 modelos de expectativa de performance.

Como um dos resultados mais importantes, os autores observaram que quando a amostra de empresas possui um desempenho diferente das firmas de controle, os métodos utilizando como benchmark a indústria, ou a indústria e o tamanho, estavam mal especificados. Por outro lado, os testes estatísticos estavam bem especificados quando as empresas da amostra de comparação possuem pré-performance similar às das empresas do grupo de controle.

A contribuição de Barber e Lyon (1996) para os estudos de eventos amparados em métricas contábeis foi no sentido de testar os diferentes modelos utilizados e apresentar aqueles que são capazes ou não de detectar performances anormais, considerando a especificidade de cada situação, e as medidas de desempenho e o modelo de expectativa operacional utilizados.

Com essa mesma preocupação de avaliar os métodos passíveis de gerar grupos de controle, para detecção da performance operacional anormal pós eventos corporativos, Lie (2001) amplia o trabalho de Barber e Lyon (1996) e testa outros modelos, utilizando grupos de controle que levem em consideração, não somente o nível de performance no passado, mas também as variações passadas nesse nível como em Benartzi, Michaely e Thaler (1997) e market-to-book - M/B como em Nohel e Tarhan (1998).

Utilizando uma amostra de 79.100 empresas entre 1978 e 1997, Lie (2001) desenvolveu 5 métodos para formar grupos de controle para detectar os retornos anormais, combinando três 
variáveis: nível de desempenho passado, variação no nível de desempenho passado e taxa M/B.

O modelo e os procedimentos estatísticos são os mesmos de Barber e Lyon (1996). Os resultados indicam que o melhor modelo para formar grupos de controle que serão utilizados como benchmark é aquele que considera o nível passado de desempenho, a sua variação, e a taxa $\mathrm{M} / \mathrm{B}$.

Porém, o autor alerta quanto à limitação de se formar grupos de controle utilizando três variáveis, pois o número de empresas desses grupos fica reduzido. Neste caso, o autor aconselha comparar pela variável nível de desempenho passado, corroborando os resultados encontrados por Barber e Lyon (1996).

Outro resultado importante dessa pesquisa é a constatação de que quando a empresa apresenta drásticas mudanças na performance operacional no passado, o ideal é controlar pelos modelos que utilizam benchmarks com empresas que também passaram por essas mudanças. E se além das variações passadas no ROA, a empresa também apresentar valores extremos do ROA, baixo ou alto, o benchmark também deve considerar o nível passado de ROA.

Esse resultado é muito relevante à medida que alguns estudos já tenham identificado nas empresas um resultado superior ao da média, como por exemplo, antes de fusões e aquisições como os de Morck et al (1990) ou IPOs como os de Jain e Kini (1994), Mikkelson, Partch, and Shah (1997), Schiozer, Oliveira e Saito (2009) e Biral (2010); Asquith; Healy e Palepu (1989) ao analisarem stock splits e Dennis e Dennis (1995) ao estudarem demissões de altos executivos. Neste sentido, Lie (2001) ressalta a importância da realização de análises preliminares com o objetivo de examinar os padrões da performance pré-evento da amostra, para só depois então escolher um método que controle as características extremas, caso elas existam.

A escolha da medida de desempenho operacional é outra questão importante na modelagem dos testes. Barber e Lyon (1996) observaram que os estudos pioneiros utilizavam a métrica lucro por ação, como o de Asquith; Healy e Palepu (1989), mas que os trabalhos recentes apresentam uma tendência na utilização do resultado operacional.

Para os autores, o resultado operacional representa uma medida mais "limpa" da produtividade dos ativos do que os lucros, pois o primeiro exclui do seu cálculo as despesas financeiras, itens especiais, depreciação, tributos e participação dos minoritários. Eles apresentam como um segundo argumento a favor da utilização do resultado operacional, em vez do lucro líquido, o fato de que geralmente os pesquisadores estão interessados em investigar eventos que resultem em mudanças na estrutura de capital e que essas mudanças afetam as despesas financeiras e consequentemente o lucro líquido, porém, não afetam o resultado operacional.

O que se pode observar é que cada trabalho argumenta para a utilização de uma métrica que mais se adapte ao fenômeno que está sendo discutido. Por exemplo, ao estudar fusões e aquisições, Healy, Palepu e Ruback (1992), Ghosh (2001) e Healy, Palepu e Ruback (1997) utilizam como métrica o Fluxo de Caixa Operacional, justificando o seu uso por ser uma medida não influenciada pelo tipo de financiamento (cash, debt ou stock acquisitions) da operação, ao contrário dos lucros que refletiriam diferenças nessas escolhas. Outro exemplo está nos estudos que abordam as Seasoned Equity Offerings - $S E O^{l}$, como os de Loughran e Ritter (1997) e o de Lukose e Rao (2003), que adotaram como medida de desempenho o EBTIDA (lucro antes de juros, impostos, depreciação e amortização).

1 Colocações primárias ou secundárias de ações feitas por companhias já listadas em bolsa. 
Baber e Lyon (1996), no entanto, alertam para as limitações do ROA, a saber, a avaliação dos ativos ao custo histórico e não ao valor de reposição ou custo corrente, o escalonamento pelo total de ativos e não apenas pelos ativos operacionais, e, por fim, a possibilidade de gerenciamento de itens que compõem o resultado operacional. Para minimizar os problemas citados, os autores utilizam as seguintes métricas alternativas: ROA ajustado ao caixa, margem operacional das vendas, retorno do valor de mercado dos ativos, e retorno do fluxo de caixa sobre ativos.

Observa-se, portanto, uma variedade de medidas de desempenho operacional em estudos que se propõem a detectar as performances anormais. E como os resultados variam dependendo da métrica utilizada, esta constitui uma importante escolha para o pesquisador, que deve considerar não só as vantagens e desvantagens de cada métrica como também a influência do evento na análise dos dados contábeis.

\section{ESTUDOS DE EVENTOS AMPARADOS EM MÉTRICAS CONTÁBEIS NO BRASIL}

Os trabalhos pioneiros no Brasil, que tratam da questão, referem-se ao desempenho das empresas após a privatização. Costa (1994), afirma que as estatais privatizadas na década de 80 apresentaram uma melhora na performance operacional, apesar de a maioria dos resultados não ser estatisticamente significativa por falta de dados.

Pinheiro (1996) examinou o impacto da privatização sobre o desempenho das estatais em dois períodos: 1981/89 e 1991/94 e adotou a mesma metodologia de Costa (1994). Os resultados foram estatisticamente significativos, indicando que o desempenho das empresas melhorou após a privatização.

Observa-se que esses dois trabalhos não utilizaram um benchmark para o controle das variáveis econômicas que afetam todas as empresas de um setor, ou de tamanho e até mesmo de pré-performance.

Eid Jr, Ribeiro e Sanvicente (2003) também investigaram a melhora da performance de 13 empresas privatizadas entre 1991 e 1998. Porém, inseriram a questão do benchmark ao adotar um grupo de controle, que neste caso foi a indústria. O teste estatístico utilizado foi o de Wilcoxon, e as medidas de desempenho a margem líquida, o ROA e o ROE. Os resultados apontaram melhora na performance operacional, com significância estatística, com relação às três medidas de desempenho analisadas.

Outros temas discutidos tratam da distribuição de dividendos e recompra de ações. Nossa e Nossa (2007) verificaram o impacto da estratégia de distribuição de dividendos e de recompra de ações na eficiência das empresas listadas na Bovespa, no período de 1995 a 2004.

A eficiência foi representada pelo ROA das empresas no ano subsequente aos eventos. As hipóteses testadas foram de que o $\operatorname{ROA}(\mathrm{t}+1)$ mediano das empresas que distribuíram dividendos ou recompraram ações no período t é estatisticamente igual ao $\mathrm{ROA}(\mathrm{t}+1)$ mediano das empresas que não distribuíram dividendos ou não recompraram ações no período $t$. 
Para o teste das hipóteses, foram utilizados o teste de normalidade, a regressão múltipla anual, bem como os testes não-paramétricos Mann-Whitney e Willcoxon.

Verificou-se que as empresas que distribuíram dividendos são mais eficientes do que as que não distribuíram. Observou-se ainda que as empresas que recompraram ações são mais eficientes do que as que não recompraram ações.

Outro estudo no mesmo ano sobre o assunto foi o de Costa Jr, Martins e Fávero (2007) sobre a influência das "poison pills" na performance das companhias abertas no Brasil. Neste trabalho, os autores buscaram testar teorias que expliquem o comportamento dos gestores com reflexo na performance operacional da firma: entrincheiramento e hipótese do interesse do acionista.

Foram analisadas 7 empresas que apresentavam "poison pills" até junho de 2006. A métrica utilizada foi EBIT dividido pelo Ativo Total. Os autores trabalharam com a hipótese nula de que não existe diferença entre as médias de Ebit anormais. Eles utilizaram tanto o teste paramétrico " $\mathrm{t}$ " quanto os não paramétricos, Mann-Whitney e teste KS, chegando à conclusão de que não há diferenças estatisticamente significativas entre as médias. Dessa forma, ambas as teorias foram refutadas para o ambiente econômico brasileiro.

Um ano depois, Costa Jr e Martins (2008) investigaram empiricamente as operações de fusão e aquisição envolvendo um total de 101 empresas listadas na BOVESPA, no período de 2002 a 2006, com a finalidade de explorar o elo existente entre as expectativas externadas pelo mercado, em decorrência das operações anunciadas, e o papel da Contabilidade na validação dessas expectativas.

O modelo utilizado pelos autores foi o do intercepto de Healy et al (1992). A métrica foi o EBITDA escalonado pelo valor contábil dos ativos totais. Foram selecionadas empresas para compor um grupo de comparação utilizando-se filtros de setor, tamanho e performance, e para este cômputo usaram tanto a média quanto a mediana. Os testes utilizados foram o teste t, Wilcoxon e de sinais. Como resultado, constatou-se a queda da performance operacional no período subsequente à operação, sendo esta diferença estatisticamente significativa. Neste sentido, ficou evidente que a Contabilidade não corroborou as expectativas médias do mercado no que concerne às possíveis sinergias advindas da combinação das empresas. Existem três trabalhos relacionados às ofertas iniciais de ações $(I P O)$, sendo que os dois primeiros foram publicados no mesmo ano.

O primeiro é o de Schiozer, Oliveira e Saito (2009) que investigaram a performance pós-IPO dos bancos brasileiros, no período de 2005 a 2007 e concluíram que existem indícios de aumento na eficiência dos bancos, indicando economias de escala resultantes da abertura de capital. Este resultado não foi estatisticamente significativo, o que de acordo com os autores, se deve à pequena amostra estudada.

Um ponto interessante apontado pelos autores é que os bancos que abriram o capital apresentaram uma pré-performance superior àqueles que permaneceram fechados. Eles também demonstraram que, apesar da performance dos bancos que realizaram o IPO ser superior antes 
e depois do evento ${ }^{2}$, esta diferença diminuiu no período pós, o que poderia ser um indicativo do efeito da reversão à média de dados contábeis.

A metodologia utilizada pelos autores foi a mesma de Jain e Kini (1994), a medida de desempenho foi o ROA, e a estatística utilizada foi o teste $t$.

O segundo trabalho é o de Bossolani e Sheng (2009) que tinham o objetivo de analisar a melhora do desempenho de 36 empresas brasileiras após o IPO, no período de 2004 a 2006. Os autores analisaram a evolução do ROE e ROA. O principal resultado encontrado foi de que não existe relevância estatística entre a diferença do retorno das empresas nos períodos pré e pós-IPO. Portanto, eles concluíram que não foi possível aceitar a hipótese de que as empresas aumentam a sua rentabilidade após o IPO.

A terceira pesquisa é a de Biral (2010) que analisou a mudança do desempenho operacional de 69 IPOs no período de 2004 a 2008. A metodologia utilizada foi a de Jain e Kini (1994).

Os testes realizados indicam que, quando comparado ao ano anterior ao IPO e controlando por setor econômico, há uma deterioração no ROA e ROE quanto ao desempenho operacional nos anos seguintes, embora não se tenha encontrado significância estatística relevante. Os resultados dos testes sem o ajuste (sem benchmark) apontam para uma melhora no desempenho.

Os resultados encontrados permitiram inferir que as empresas que abriram capital eram mais eficientes antes do IPO, quando comparadas a empresas de capital aberto do mesmo setor e tamanho semelhante, não sendo possível identificar se houve mudança de desempenho operacional pós-IPO.

\section{CONSIDERAÇÕES FINAIS}

Até o momento, com exceção de alguns poucos trabalhos, observa-se o seguinte dominador comum entre as pesquisas que utilizaram a técnica de estudo de eventos amparado em métricas contábeis, realizadas no Brasil: resultados estatisticamente inconclusivos, o que em alguns casos pode ser justificado pela utilização de pequenas amostras.

Outro ponto em comum nos estudos brasileiros é que nenhum deles, com exceção de Costa Jr e Martins (2008), utilizou o benchmark pré-performance, tão destacado por Barber e Lyon (1996), Lie (2001), como variável fundamental para a formação do grupo de controle, especialmente quando as empresas amostradas apresentavam pré-desempenho diferente das do grupo de controle, uma questão observada por Schiozer, Oliveira e Saito (2009) e Biral (2010).

Nota-se também a preferência dos autores brasileiros em utilizar como medida de performance o ROA e ROE, colocando no numerador o lucro líquido, que, conforme já apontado por Barber e Lyon (1996), apresenta problemas de mensuração quando são incluídas variáveis que podem ser afetadas pela forma de financiamento da operação, como as despesas

2 Uma medida que também é documentada na literatura reside no consenso de analistas sell-side acerca do lucro esperado de uma companhia. Contudo, conforme também documentado na literatura, analistas sell-side costumam ser excessivamente otimistas. 
financeiras, por exemplo, e que não retratam a realidade operacional.

Por fim, outro aspecto relevante se destaca: os estudos de eventos baseados em métricas contábeis no Brasil são recentes e em menor número quando comparados à literatura internacional, o que se justifica pelo recente desenvolvimento do mercado de capitais brasileiros, abertura e estabilização econômicas. Diante do cenário apresentado acima, destaca-se que existe um espaço para este tipo de pesquisa envolvendo eventos corporativos com empresas brasileiras.

\section{REFERÊNCIAS}

ASQUITH, Paul; HEALY, Paul e PALEPU, Krishna. Earnings and stock splits. The Accounting Review, v. 64, n. 3, p. 387-403, jul., 1989.

BALL R.; BROWN, P. An Empirical Eavaluation of Accounting Income Numbers. Journal of Accounting Research, Chicago, v. 6, n.2, p. 159-178, 1968.

BARBER, B.M.; LYON, J.D. Detecting Abnormal Operating Performance: The Empirical Power and Specification of Test Statistics. Journal of Financial Economics, North-Holland, v.41, p. 359-399, 1996.

BENARTZI, S., MICHAELY, R. e THALER, R. Do Changes in Dividends Signal the Future or the Past? Journal of Finance, v. 52, n.3, p.1007-1034, jan., 1997.

BIRAL, Rafael Augusto Fernandes. A abertura de capital afeta o desempenho operacional das empresas? Evidência da onda de IPOs 2004-2008. Escola de Economia de São Paulo, Fundação Getúlio Vargas. São Paulo, 2010, 50 p.

BOSSOLANI, Tharso; SHENG, Hsia Hua. Oferta Inicial de Açoes e o Desempenho das Empresas. Anais... In: XXXIII Encontro da ANPAD, São Paulo-SP, 2009. CD-ROM.

COSTA, C. Uma análise dos impactos da privatização sobre o desempenho das empresas alienadas na década de 80. Rio de Janeiro: FEA/UFRJ, 1994 (Texto Discente, 20)

COSTA JUNIOR, J.V. Retornos anormais versus performances operacionais anormais de firmas brasileiras envolvidas em fusões e aquisições no período de 2002 a 2006. Faculdade de Economia, Administração e Contabilidade da Universidade de São Paulo, São Paulo, 2008, 155 p.

COSTA JUNIOR, Jorge Vieira; MARTINS, Eliseu. Retornos Anormais versus Performances Operacionais Anormais de Firmas Brasileiras Envolvidas em Fusões e Aquisições no Período de 2002 a 2006. Anais... In: XXXII Encontro da ANPAD, Rio de Janeiro-RJ, 2008. CD-ROM.

COSTA JUNIOR, Jorge Vieira; MARTINS, Eliseu; FÁVERO, Luiz Paulo. As "Poison Pills" Influenciam a Performance Operacional das Companhias Abertas no Brasil? Anais... In: XXXI Encontro da ANPAD, Rio de Janeiro-RJ, 2007. CD-ROM

DANN, Larry; MASULIS, Ron e MAYERS, David. Repurchase tender offers and earnings information. Journal of Accounting and Economics, v. 14, p. 217-251, 1991. 
DEGEORGE, Francois e ZECKHAUSER, Richard. The reverse LBO decision and firm performance: Theory and evidence. Journal of Finance, v. 48, n. 4, p. 1323-1348, setembro, 1993.

DENIS, David J. e DENIS, Diane K. Managerical discretion, organization structure e corporate performance: A study of levareged recapitalizations. Journal of Accounting and Economics, v. 16, p. 209-236, 1993.

DENIS, David J. e DENIS, Diane K. Performance Changes Following Top Management Dismissals. Journal of Finance, v.50, n. 4, p. 1029-1057, Set., 1995.

EID JUNIOR, William; RIBEIRO, Marcos Poplawski; SANVICENTE, Antonio Zoratto. Análise do Desempenho Financeiro e Operacional das Empresas Recentemente Privatizadas no Brasil. Anais... In: III Encontro Brasileiro de Finanças, São Paulo, SP, 2003.

FAMA E. F. et al. The Adjustment of Stock Prices to new information. International Economic Review, Pennsylvania, v. 10, n.1, p. 1-21, Fev, 1969.

FAMA, Eugene. F.; FRENCH, K. R. Forecasting Profitability and Earnings. Journal of Business, n. 73, p. 161-175, 2000.

FAMA, Eugene F.; FRENCH, Kenneth R. Size and book-to-market factors in earnings and returns. Journal of Finance, v. 50, n.1, p. 131-155, março, 1995.

GHOSH, A. Does Operating Performance really Improve following Corporate Acquisitions? Journal of Corporate Finance, [S.1.], v. 7, n.2, p. 151-178, junho, 2001.

GONZÁLES, Marcelo; FARIAS, Pablo. Desempeño Operacional Posterior a la Oferta Pública Inicial de Acciones de Las Empresas Chilenas. El Trimestre Económico. Tlalpan: Jul-Set, v. 76, n. 3, p. 751-774, 2009.

GUO, Shourun, HOTCHKISS, Edith S.; SONG, Weihong. Do Buyouts (Still) Create Value? Journal of Finance, Forthcoming, Agosto, 2009. Disponível em: http://ssrn.com/ abstract=1009281 Acesso em: 12 mai. 2010.

HEALY, P.; PALEPU, K. Earnings Information Conveyed by Dividend Initiations. Journal of Financial Economics, n. 21, p. 149-175, 1988.

HEALY, P.M.; PALEPU; K.G.; RUBACK, R.S. Does corporate performance improve after mergers? Journal of Financial Economics, v. 31, p. 135-175, 1992.

HEALY, Paul; PALEPU, Krishna e RUBACK, Richard. Which takeovers are profitable Strategic or Financial? Sloan Management Review, v. 38, n. 4, p. 45-57, 1997.

JAIN, Bharat, A.; KINI Omesh. The post-issue operating performance of IPO firms. Journal of Finance, Cambridge, v. 49, n.5, p. 1699-1726, dezembro, 1994.

KAPLAN, Steven. The effect of management buyouts on operating performance and value. Journal of Financial Economics, Boston, v. 24, n.2, p. 217-254, Setembro, 1989.

LIE, Erik, Detecting Abnormal Operating Performance: Revisited. Financial Management, Vol. 30, n. 2, Jun, 2001. 
LOUGHRAN, Tim; RITTER, Jay. The operating performance of firms conducting seasoned equity offerings. Journal of Finance, v. 32, n.5, dezembro, 1997.

LUKOSE, Jijo e RAO, S. Narayan, Operating Performance of the Firms Issuing Equity through Rights Offer. Junho, 2003. Disponível em: http://ssrn.com/abstract=428102 . Acesso em: 15 mai. 2010.

MIKKELSON, W. H.; PARTCH, M. M.; SHAH, K. Ownership and Operating Performance of Companies that Go Public. Journal of Financial Economics, 44, 281-307, 1997.

MORCK, R.; SHLEIFER, A.; VISHNY, R.W. Do managerial objectives drive bad acquisitions? Journal of Finance, v. 45, p. 31-48, 1990.

NOHEL, T. and V. TARHAN. Share Repurchases and Firm Performance: New Evidence on the Agency Costs of Free Cash Flow. Journal of Financial Economics v. 49, n. 2, p. 187-222, ago., 1998.

NOSSA, Valcemiro; NOSSA, Silvania Neris. O impacto da Distribuição de dividendos e da Recompra de ações na eficiência das empresas listadas na Bovespa (1995 a 2004). Anais... In: XXXI Encontro da ANPAD, Rio de Janeiro-RJ, 2007. CD-ROM.

PINHEIRO, A. C. Impactos Microeconômicos da Privatização no Brasil. Pesquisa e Planejamento Econômico, v. 26, n.3, p. 357-397, 1996.

SCHIOZER, R.F., OLIVEIRA, R.F. e SAITO, R. Why Banks go Public? Evidence from the 2005-2007 Wave of Brazilian Bank-IPO. Working Paper, 2009. Disponivel em http://ssrn.com/ abstract=1470994. Acesso em: 15 jun. 2010. 


\section{DADOS DOS AUTORES:}

\section{MELISA MAIA DE PAULA}

Universidade do Estado do Rio de Janeiro - UERJ

Rua São Francisco Xavier, 524, $9^{\circ}$ andar, Bloco E, Maracanã,

20550-013 - Rio de Janeiro, RJ - Brasil

\section{JORGE VIEIRA}

Universidade do Estado do Rio de Janeiro

Departamento de Contabilidade - Faculdade de Administração e Finanças/FAF

Rua São Francisco Xavier, 524, $9^{\circ}$ andar, Bloco E, Maracanã,

20550-013 - Rio de Janeiro, RJ - Brasil 